\title{
NLO PDFs from the ABMP16 fit
}

\author{
S. Alekhin ${ }^{1,2}$, J. Blümlein ${ }^{3}$, S. Moch ${ }^{1, a}$ \\ ${ }^{1}$ II. Institut für Theoretische Physik, Universität Hamburg, Luruper Chaussee 149, 22761 Hamburg, Germany \\ ${ }^{2}$ Institute for High Energy Physics, Protvino, Moscow 142281, Russia \\ ${ }^{3}$ Deutsches Elektronensynchrotron DESY, Platanenallee 6, 15738 Zeuthen, Germany
}

Received: 22 March 2018 / Accepted: 29 May 2018 / Published online: 11 June 2018

(C) The Author(s) 2018

\begin{abstract}
We perform a global fit of parton distribution functions (PDFs) together with the strong coupling constant $\alpha_{s}$ and the quark masses $m_{c}, m_{b}$ and $m_{t}$ at next-to-leading order (NLO) in QCD. The analysis applies the $\overline{\mathrm{MS}}$ renormalization scheme for $\alpha_{s}$ and all quark masses. It is performed in the fixed-flavor number scheme for $n_{f}=3,4,5$ and uses the same data as the previous ABMP16 fit at next-to-nextto-leading order (NNLO). The new NLO PDFs complement the set of ABMP16 PDFs and are to be used consistently with NLO QCD predictions for hard scattering processes. At NLO we obtain the value $\alpha_{s}^{\left(n_{f}=5\right)}\left(M_{Z}\right)=0.1191 \pm 0.0011$ compared to $\alpha_{s}^{\left(n_{f}=5\right)}\left(M_{Z}\right)=0.1147 \pm 0.0008$ at NNLO.
\end{abstract}

Parton distribution functions (PDFs) are an indispensable ingredient in theory predictions for hadronic scattering processes within perturbative QCD. Currently, the state-of-art calculations for many standard-candle processes at the Large Hadron Collider (LHC) and elsewhere are based on the QCD corrections up to the next-to-next-to-leading order (NNLO) in the strong coupling constant $\alpha_{s}$ [1]. In order to match this theoretical accuracy the PDFs and other input parameters such as $\alpha_{s}$ and the quark masses $m_{c}, m_{b}$ and $m_{t}$ also have to be determined at the same order of perturbation theory, that is with account of the NNLO QCD corrections. In many instances, however, the Wilson coefficient functions or hard partonic scattering cross sections are known to the next-to-leading order (NLO) only. This concerns in particular Monte-Carlo studies at the LHC. Then, to meet the consistency requirements, NLO PDFs and the respective NLO values for $\alpha_{s}$ and the heavy-quark masses are to be used. The NLO fit of PDFs is therefore of immediate practical use and also provides a very good consistency check of the perturbative stability of QCD calculations.

In this article we describe the NLO version of the recent ABMP16 PDF fit, i.e., the NLO analysis, which applies the $\overline{\mathrm{MS}}$ scheme for $\alpha_{s}$ and all heavy-quark masses. It uses the

a e-mail: sven-olaf.moch@desy.de same data, their uncertainty treatment and the general theoretical framework, e.g., the fixed-flavor number scheme for $n_{f}=3,4$ and 5, as in the previous fit of the ABMP16 PDFs NNLO. The only difference resides in the order of the perturbative corrections to the QCD evolution equations and for the Wilson coefficients, which are now limited to NLO accuracy. Due to the obvious correlations of the various parameters in the PDFs with the value of $\alpha_{s}$ and those of the quark masses $m_{c}, m_{b}$ and $m_{t}$, all quantities are extracted simultaneously from the global fit following our previous analyses [2-4]. The article discusses in detail the differences in their determinations at NLO and NNLO accuracy. Specific attention is paid to the treatment of power corrections in the description of deep-inelastic scattering (DIS) data, i.e. higher-twist effects which are relevant beyond the leading twist collinear factorization approximation. The final fit results are made available as data grids for use with the LHAPDF library (version 6) [5] and the features of the various grids are briefly discussed.

The values of $\chi^{2}$ obtained in the present analysis for various data sets are listed in Table 1 in comparison with the earlier ones for the NNLO ABMB16 fit. The overall quality of the data description does not change dramatically between the NLO and the NNLO versions, where the former features a somewhat bigger total value of $\chi^{2}$. Of course, the theoretical description at NNLO accuracy comes with a significantly reduced theoretical uncertainty due to variations of the factorization and renormalization scales compared to the NLO one. Nevertheless, for specific scattering reactions the NNLO corrections are crucial for the respective data sets. This holds in particular for the $c$-quark and, to a lesser extent, for $b$-quark production in DIS and for hadronic $t$-quark pair-production, which constrain the heavy-quark masses and which are fitted together with $\alpha_{s}$ simultaneously with the PDFs. The theoretical description at NNLO accuracy is also essential for the parameters of the higher (dynamical) twist, which contribute additively to the leading twist. The $x$-dependent twist-four contributions to the longitudinal and transverse DIS cross 
Table 1 The values of $\chi^{2}$ obtained in the present analysis at NLO for the data on inclusive DIS, the DY process, and on heavy-quark production in comparison with the ones of the ABMP16 fit at NNLO [4]. 'This value corrects a misprint in Table V of Ref. [4]

\begin{tabular}{|c|c|c|c|c|}
\hline \multirow[t]{2}{*}{ Experiment } & \multirow[t]{2}{*}{ Process } & \multirow[t]{2}{*}{ NDP } & \multicolumn{2}{|l|}{$\chi^{2}$} \\
\hline & & & NLO & NNLO \\
\hline \multicolumn{5}{|l|}{ DIS } \\
\hline HERA I+II & $\begin{array}{l}e^{ \pm} p \rightarrow e^{ \pm} X \\
e^{ \pm} p \rightarrow \stackrel{(-)}{v} X\end{array}$ & 1168 & 1528 & 1510 \\
\hline Fixed-target (BCDMS, NMC, SLAC) & $l^{ \pm} p \rightarrow l^{ \pm} X$ & 1008 & 1176 & 1145 \\
\hline \multicolumn{5}{|l|}{ DIS heavy-quark production } \\
\hline HERA I+II & $e^{ \pm} p \rightarrow e^{ \pm} c X$ & 52 & 58 & $66^{a}$ \\
\hline H1, ZEUS & $e^{ \pm} p \rightarrow e^{ \pm} b X$ & 29 & 21 & 21 \\
\hline Fixed-target (CCFR, CHORUS, NOMAD, NuTeV) & $\stackrel{(-)}{v} N \rightarrow \mu^{ \pm} c X$ & 232 & 173 & 178 \\
\hline \multicolumn{5}{|l|}{ DY } \\
\hline ATLAS, CMS, LHCb & $\begin{array}{l}p p \rightarrow W^{ \pm} X \\
p p \rightarrow Z X\end{array}$ & 172 & 229 & 223 \\
\hline Fixed-target (FNAL-605, FNAL-866) & $p N \rightarrow \mu^{+} \mu^{-} X$ & 158 & 219 & 218 \\
\hline \multicolumn{5}{|l|}{ Top-quark production } \\
\hline ATLAS, CMS & $p p \rightarrow t q X$ & 10 & 5.7 & 2.3 \\
\hline CDF\&DØ & $\begin{array}{l}\bar{p} p \rightarrow t b X \\
\bar{p} p \rightarrow t q X\end{array}$ & 2 & 1.9 & 1.1 \\
\hline ATLAS, CMS & $p p \rightarrow t \bar{t} X$ & 23 & 14 & 13 \\
\hline $\mathrm{CDF} \& \mathrm{D} \varnothing$ & $\bar{p} p \rightarrow t \bar{t} X$ & 1 & 1.4 & 0.2 \\
\hline Total & & 2855 & 3427 & 3378 \\
\hline
\end{tabular}

sections have been determined in the NNLO version (cf. Table VIII in Ref. [4]) and their central values are kept fixed in the present analysis. Also other fitted parameters like the data set normalizations are taken over unchanged from the NNLO analysis (cf. Table I in Ref. [4]). This provides a better consistency between the PDF sets obtained with different theoretical accuracy. At the same time the uncertainties in the normalization and higher twist parameters are computed in the same way as in the NNLO fit [4], by propagation of the ones in experimental data and simultaneosly with other fit parameters in order to take into account their correlations and, therefore, provide a consistent uncertainty treatment in the NLO and NNLO fits. Therefore, the uncertainties obtained for the data normalization and the twist-four contributions at NLO are only marginally different from those reported at NNLO in Tables I and VIII in Ref. [4].

Closer inspection of the $\chi^{2}$-values in Table 1 reveals the largest differences between NLO and NNLO for the fixedtarget DIS data, which can be explained by the kinematic coverage of this data sample, which is predominantly in the low- $Q^{2}$ region. For the Drell-Yan (DY) data the impact of the NNLO QCD corrections is less pronounced and the corresponding improvement in the value of $\chi^{2}$ is small. For the heavy-quark production data on the other hand the trend is not uniform, i.e., for some data sets the $\chi^{2}$-values at NLO are larger and vice versa for others. In this context it is worth noting, that to a certain extent the impact of missing NNLO terms in the NLO fit is compensated by tuning the values of heavy-quark masses.

The ABMP16 PDF sets at NLO and NNLO are compared in Fig. 1 for the case of $n_{f}=3$ flavors at the scale $\mu=3 \mathrm{GeV}$. Both sets are based on the same flexible parametrization used in Ref. [4]. For the gluon PDF, we see in Fig. 1a that the NLO PDFs are larger by about $15 \%$ in the small- $x$ and the large- $x$ region, i.e., for $x \lesssim 10^{-4}$ and $x \gtrsim 0.3$, respectively. In these kinematic regions for example the DIS coefficient functions receive systematically large corrections at higher orders, which need to be compensated by the gluon PDF if the fit is performed at NLO accuracy. The $u$-quark PDF in Fig. 1b does not show any big changes, except for large $x \gtrsim 0.6$, while the $d$-quark PDF Fig. $1 \mathrm{c}$ at NLO is smaller in the entire range $x \lesssim 10^{-1}$ and decreasing more than $20 \%$ for $x \lesssim 10^{-4}$. A similar observation holds for the strange sea displayed in Fig. 1d, which is smaller by even $50 \%$ for $x \lesssim 10^{-4}$ at NLO, however, the PDF uncertainties for this quantity are correspondingly larger. On the other hand, the non-strange sea in Fig. 1e does not show big relative differences between NLO and NNLO. There is only a slight decrease of the NLO result by $5 \%$ to $10 \%$ for $x \lesssim 10^{-2}$. The small- $x$ sea iso-spin asymmetry $\bar{d}-\bar{u}$ at NLO goes lower than the NNLO one, as 


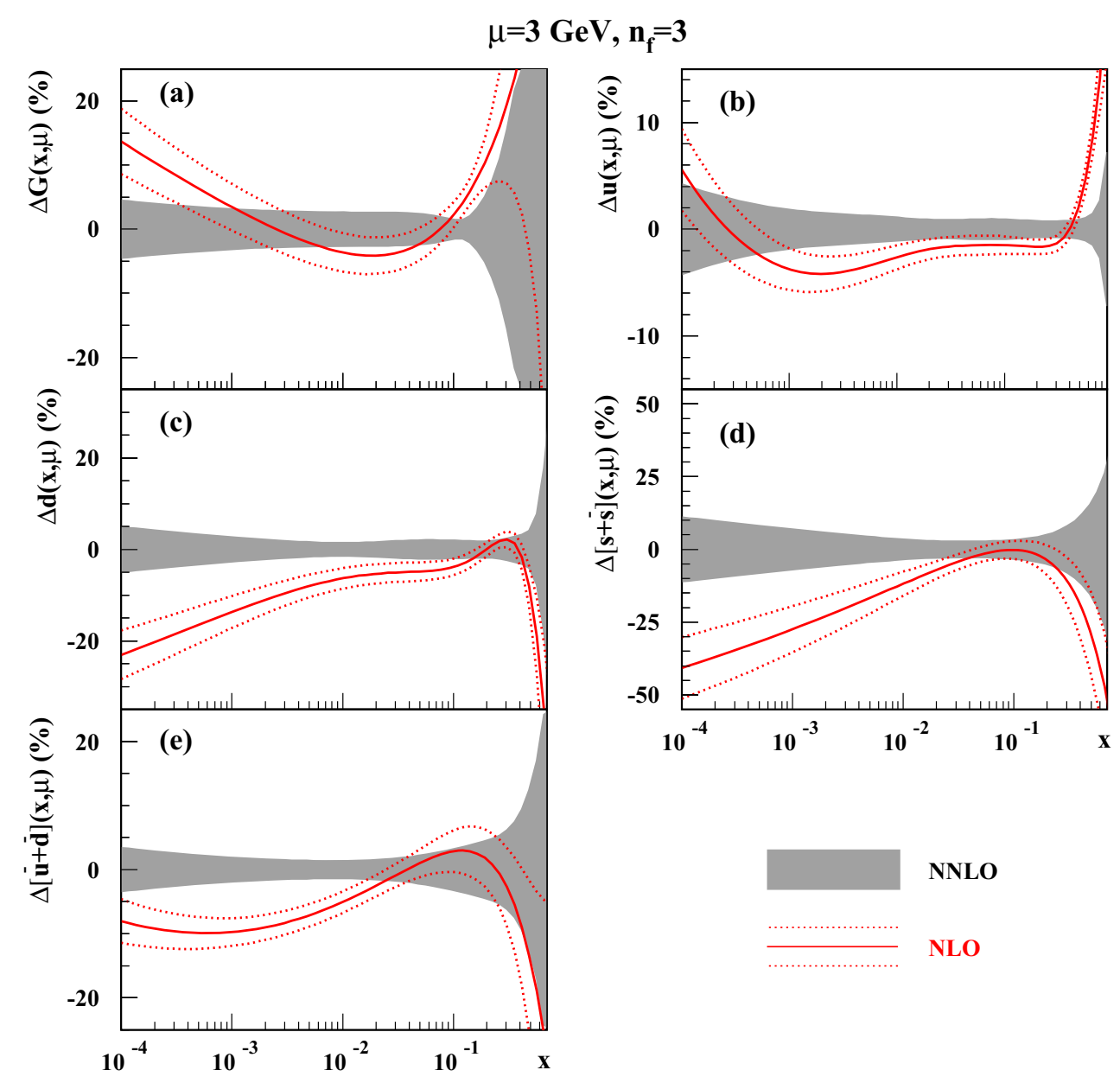

Fig. 1 The $1 \sigma$ band for the $n_{f}=3$ flavor NNLO ABM16 PDFs [4] for $\mathbf{a}$ gluon, $\mathbf{b}$ up-quarks $\mathbf{c}$ down-quarks $\mathbf{d}$ the symmetrized strange sea and $\mathbf{e}$ the non-strange sea, at the scale of $\mu=3 \mathrm{GeV}$ versus $x$ (shaded

area) compared with the relative difference of those PDFs to the NLO ABMP16 ones obtained in the present analysis (solid lines). The dotted lines display $1 \sigma$ band for the NLO PDFs

can be seen from a comparison of Fig. 1b, c. This reflects the impact of the NNLO corrections on the data for Drell-Yan production, which drive this asymmetry in our fit.

The value of $\alpha_{s}\left(M_{Z}\right)$ at the scale of the $Z$-boson mass $M_{Z}$ obtained in the present analysis at NLO is larger than the one obtained in the NNLO variant of the ABMP16 fit [4], the relative difference amounting to about $4 \%$, which is well comparable to the estimated margin due to variations of the factorization and renormalization scales, cf. Ref. [6]. In the scheme with $n_{f}=5$ light flavors we find

$$
\begin{aligned}
\alpha_{s}^{\mathrm{NLO}}\left(M_{Z}\right) & =0.1191 \pm 0.0011, \\
\alpha_{s}^{\mathrm{NNLO}}\left(M_{Z}\right) & =0.1147 \pm 0.0008,
\end{aligned}
$$

as listed in the first line of Table 2 together with the kinematic cuts imposed on the DIS data. The description of DIS data at those low values of $Q^{2}$ and $W$ for the invariant mass of the hadronic system, where $W^{2}=M_{P}^{2}+Q^{2}(1-x) / x$ with the proton mass $M_{P}$, requires modeling of the higher-twist terms. This has been discussed extensively in the ABMP16 analyses at NNLO [4]. Following the theoretical framework there, the fitted twist-four contributions to the longitudinal and transverse DIS cross sections have been used to determine the value of $\alpha_{s}^{\mathrm{NLO}}\left(M_{Z}\right)$ in Eq. (1). Alternatively, one can impose cuts both on $Q^{2}$ and $W^{2}$ to eliminate data from the kinematic regions most sensitive to the higher-twist terms. Then, the fit can be performed with all higher-twist terms set to zero and the results are shown in Table 2. These variants of the fit with substantially higher cuts on $Q^{2}$ and $W^{2}$ and higher-twist terms set to zero display very good stability of the value of $\alpha_{s}\left(M_{Z}\right)$, both at NLO and NNLO, and therefore very good consistency of the chosen approach.

Finally, in one of the variants of the present analysis we impose the low cuts on $Q^{2}$ and $W^{2}$ from the first line of Table 2, while fitting also the twist-four contributions. This gives an improvement in the value of $\chi^{2}$ equal to 86 and $\alpha_{s}^{\mathrm{NLO}}\left(M_{Z}\right)=0.1227 \pm 0.0011$, which is a slightly larger value than those quoted in Table 2 for the fits with higher cuts on $Q^{2}$ and $W^{2}$. The magnitude of these shifts in $\alpha_{s}\left(M_{Z}\right)$ 
Table 2 The values of $\alpha_{s}\left(M_{Z}\right)$ obtained in the NLO and NNLO variants of the ABMP16 fit with various kinematic cuts on the DIS data imposed and different modeling of the higher twist terms

\begin{tabular}{llll}
\hline & Fit ansatz & \multicolumn{2}{c}{$\alpha_{s}\left(M_{Z}\right)$} \\
\hline Higher twist modeling & Cuts on DIS data & NLO & NNLO \\
Higher twist fitted & $Q^{2}>2.5 \mathrm{GeV}^{2}, W>1.8 \mathrm{GeV}$ & $0.1191(11)$ & $0.1147(8)$ \\
& $Q^{2}>10 \mathrm{GeV}^{2}, W^{2}>12.5 \mathrm{GeV}^{2}$ & $0.1212(9)$ & $0.1153(8)$ \\
Higher twist fixed at 0 & $Q^{2}>15 \mathrm{GeV}^{2}, W^{2}>12.5 \mathrm{GeV}^{2}$ & $0.1201(11)$ & $0.1141(10)$ \\
& $Q^{2}>25 \mathrm{GeV}^{2}, W^{2}>12.5 \mathrm{GeV}^{2}$ & $0.1208(13)$ & $0.1138(11)$ \\
\hline
\end{tabular}

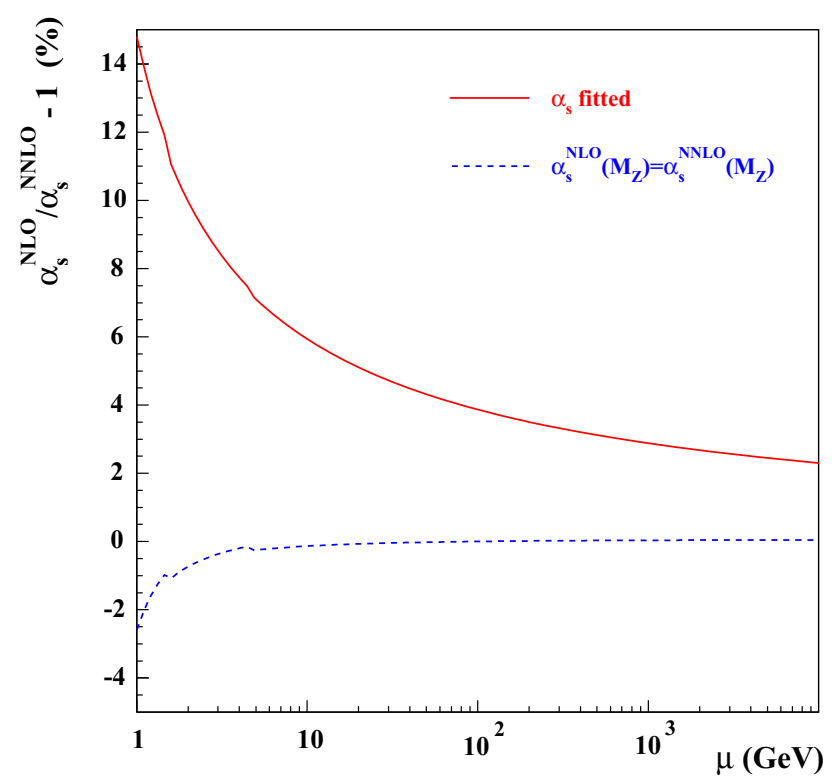

Fig. 2 The relative difference between $\alpha_{s}^{\mathrm{NLO}}(\mu)$ and $\alpha_{s}^{\mathrm{NNLO}}(\mu)$ as a function of the renormalization scale $\mu$. The solid line denotes the results of the present analysis (NLO) and of Ref. [4] (NNLO), the dashed line displays the ones derived using matching $\alpha_{s}^{\mathrm{NLO}}=\alpha_{s}^{\mathrm{NNLO}}$ at the scale $M_{Z}$

may also be considered as an indication for the limitations of the NLO approximation.

Nevertheless, the observed difference between $\alpha_{s}^{\mathrm{NLO}}(\mu)$ and $\alpha_{s}^{\mathrm{NNLO}}(\mu)$ is quite essential, particularly at small scales $\mu$, where the NLO and NNLO results differ by more than 10\%, as illustrated in Fig. 2 for a wide range of scales. This difference is to a great extent responsible for the perturbative stability of QCD calculations at the hard scales currently probed in scattering processes at colliders. Asymptotic freedom in QCD, i.e. stability of theoretical predictions under higher order perturbative corrections requires very large scales. On the other hand, for realistic kinematics including experiments at the LHC a consistent setting of $\alpha_{s}\left(M_{Z}\right)$ is very important to achieve sensible theoretical predictions. ${ }^{1}$

\footnotetext{
$\overline{1}$ Recent reviews on determinations of $\alpha_{s}\left(M_{Z}\right)$, particularly those involving PDF fits, can be found in [7], in Sect. 4 of Ref. [1] and in Sect. III.D of Ref. [4]. Determinations of $\alpha_{s}\left(M_{Z}\right)$ in DIS and including jet cross section measurements have been discussed in Ref. [8].
}

Table 3 The values of the $c$-, $b$ - and $t$-quark masses in the $\overline{\mathrm{MS}}$ scheme in units of $\mathrm{GeV}$ obtained in the NLO and NNLO variants of the ABMP16 fi. The quoted errors reflect the uncertainties in the analyzed data

\begin{tabular}{lcc}
\hline & NLO & NNLO \\
\hline$m_{c}\left(m_{c}\right)[\mathrm{GeV}]$ & $1.175 \pm 0.033$ & $1.252 \pm 0.018$ \\
$m_{b}\left(m_{b}\right)[\mathrm{GeV}]$ & $3.88 \pm 0.13$ & $3.84 \pm 0.12$ \\
$m_{t}\left(m_{t}\right)[\mathrm{GeV}]$ & $162.1 \pm 1.0$ & $160.9 \pm 1.1$ \\
\hline
\end{tabular}

In this context it is worth to mention the conventional choice $\alpha_{s}^{\mathrm{NLO}}\left(M_{Z}\right)=\alpha_{s}^{\mathrm{NNLO}}\left(M_{Z}\right)$, which is adopted as a part of the PDF4LHC recommendations [9] and employed in the CT14 [10] and NNPDF [11] PDF fits. Under this assumption the value of $\alpha_{s}$ obtained at NLO is very close to the NNLO one in a wide range of scales, as shown in Fig. 2. As a result, such an approach has significant limitations when studying the convergence of the perturbative expansion, since the NLO predictions obtained with these PDF sets might be very similar to the NNLO ones simply due to the convention used.

The values for the heavy-quark masses obtained in the NLO and NNLO variants of the ABMP16 analysis are given in Table 3. The comparison of the difference between these values indicates again the limitations of the NLO approximation. The shifts are more significant for $m_{c}$ and $m_{t}$, since the NNLO corrections are absolutely essential in order to achieve a good description of the data on DIS $c$-quark production and $t$-quark hadro-production. On the other hand, the data on DIS $b$-quark production are less precise, therefore the value of $m_{b}$ extracted from the fit suffers from the larger uncertainties and is less sensitive to the impact of the NNLO corrections, cf. Table 3.

In addition, the value of $m_{t}$ demonstrates a strong correlation with the value of $\alpha_{s}$, since the Born cross section for $t \bar{t}$ production is proportional to $\alpha_{s}^{2}$, so that changes in the value of $\alpha_{s}$ induce shifts in the fitted value of $m_{t}[3,4]$. This is quantified in Fig. 3, where the values of $m_{t}$ determined in variants of the present analysis with fixed values of $\alpha_{s}$ demonstrate a nearly linear dependence on $\alpha_{s}$. It is interesting to note, though, that at NLO this dependence is somewhat shallower than for the similar fit at NNLO since due to important missing QCD corrections of $\mathcal{O}\left(\alpha_{s}^{4}\right)$ in the hadronic $t \bar{t}$-production cross section at NLO, $\sigma(t \bar{t})$ is less sensitive to the $\alpha_{s}$ vari- 


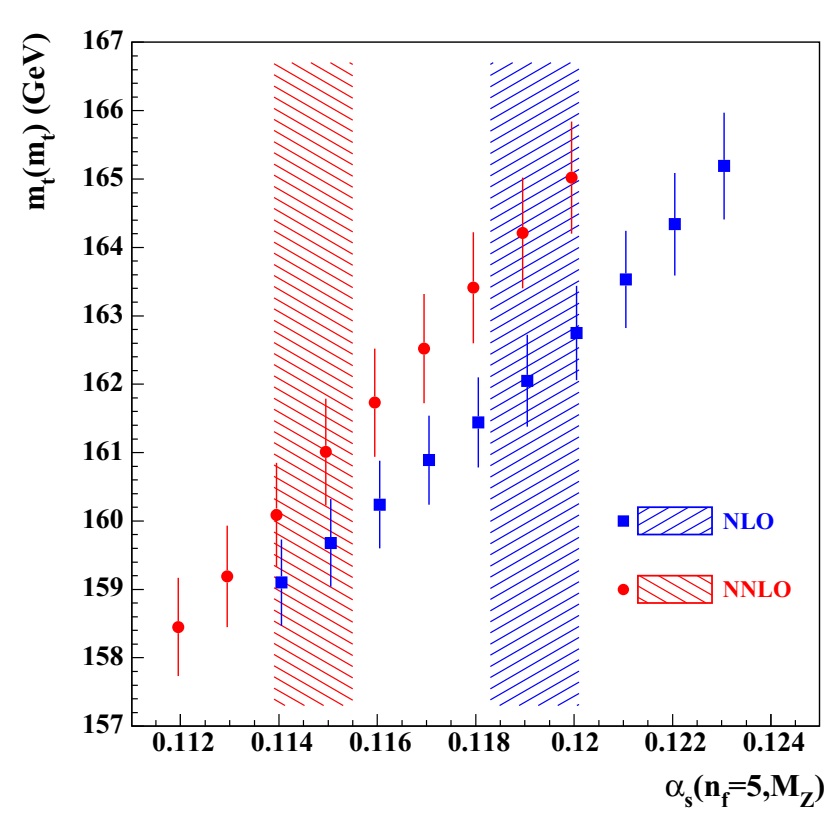

Fig. 3 The $\overline{\mathrm{MS}}$ values of the $t$-quark mass $m_{t}\left(m_{t}\right)$ obtained in the variants of the NLO ABMP16 fit with $\alpha_{s}^{\left(n_{f}=5\right)}\left(M_{Z}\right)$ fixed (squares) in comparison to ones at NNLO (circles). The left-tilted and right-tilted hatch represent the $1 \sigma$ bands for $\alpha_{s}^{\left(n_{f}=5\right)}\left(M_{Z}\right)$ obtained in the ABMP16 nominal fits at NLO and NNLO, respectively. The points are slightly shifted left and right to prevent overlapping

ations at this order. For the same reason the NLO value of $m_{t}$ is substantially larger than the NNLO one. This comparison demonstrates the necessity for a consistent treatment of the higher order corrections to the hard partonic scattering together with the parameter choices for $\alpha_{s}$ and $m_{t}$, especially in analysis of the $t \bar{t}$-production data. To that end, the PDFs from the variants entering Fig. 3 with the a fixed value of $\alpha_{S}\left(M_{Z}\right)$ in the range of $0.114 \div 0.123$ are made available as well. It should be stressed, though, that those PDFs at preselected values of $\alpha_{s}\left(M_{Z}\right)$ are not providing the $\chi^{2}$ minimum in the analysis of all the data.

In contrast, the determination of the charm-quark mass $m_{c}$ is not very sensitive to the value of $\alpha_{s}$ because the variation of $\alpha_{s}$ is compensated by a change in the gluon distribution at small $x$ [12] (cf. also Table B in Ref. [4] for correlations between $\alpha_{S}$ and the quark masses). Indeed, the fitted value of $m_{c}$ only changes by $\pm 20 \mathrm{MeV}$ for a variation of $\alpha_{s}$ in a wide range, cf. Fig. 4. However, the NNLO corrections to the Wilson coefficents for DIS heavy-quark production still have significant impact on $m_{c}$, moving it up by $\sim 100 \mathrm{MeV}$ and reducing its uncertainty. The relatively weak correlation of $m_{c}$ with $\alpha_{s}$ in Fig. 4 is in contrast to the observed behavior in the MMHT14 PDF set [13], where the particular variable flavor number scheme applied causes a linear relation between $m_{c}$ and $\alpha_{s}$ in those fits and the charm-quark mass has been treated as a variable parameter, while the resulting values of $\chi^{2}$ when fitting data on DIS $c$-quark production have been quantified. See Sect. 3.2 and Tables 4 and 5 in Ref. [1] for

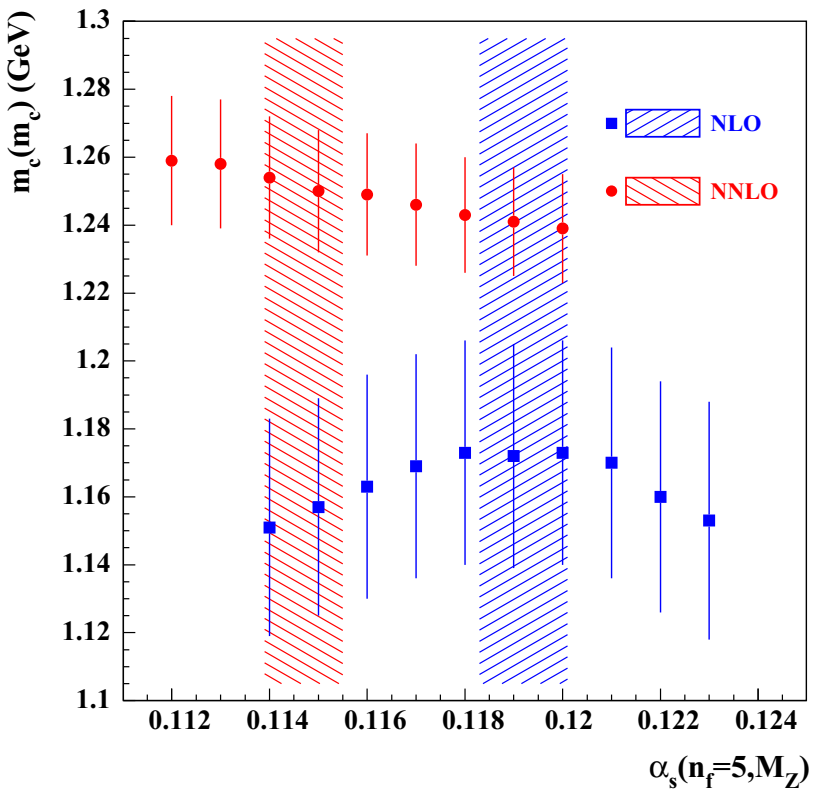

Fig. 4 The same as in Fig. 3 for the $\overline{\mathrm{MS}}$ value of the $c$-quark mass $m_{c}\left(m_{c}\right)$

a review of the theoretical treatments of DIS $c$-quark production used in PDF fits and Table 2 in Ref. [13] as well as Table 12 in Ref. [1] for the respective values of $m_{c}, \alpha_{s}$ and $\chi^{2}$ in the MMHT14 PDF set.

The grids for the NLO PDFs obtained in the present analysis are accessible with the LHAPDF library (version 6) [5] and available for download under http://projects . hepforge.org/lhapdf. For a fixed number of flavors, $n_{f}=3,4$ and 5 , we provide

ABMP16_3_nlo $(0+29)$,
ABMP16_4_nlo $(0+29)$,
ABMP16_5_nlo $(0+29)$,

which consist of the central fit (set 0 ) and additional 29 sets for the combined symmetric uncertainties in all parameters (PDFs, $\alpha_{s}, m_{c}, m_{b}$ and $m_{t}$ ). In each PDF set, the strong coupling $\alpha_{s}$ is taken in the corresponding scheme, i.e., $\alpha_{s}^{\left(n_{f}=3\right)}$, $\alpha_{s}^{\left(n_{f}=4\right)}$ and $\alpha_{s}^{\left(n_{f}=5\right)}$ which can be related by the standard decoupling relations in QCD. As usual, the PDF set with three light-quarks $n_{f}=3$, ABMP16_3_nlo, is valid at all perturbative scales $\mu^{2} \gtrsim 1 \mathrm{GeV}^{2}$, while those with $n_{f}=4$ and $n_{f}=5$, ABMP16_4_nlo and ABMP16_5_nlo, are subject to minimal cuts in $\mu^{2} \geq 3 \mathrm{GeV}^{2}$ and $\mu^{2} \geq 20 \mathrm{GeV}^{2}$, respectively, cf. Ref. [4] for additional discussions.

For studies of LHC observables and their dependence on $\alpha_{s}$ we also provide NLO PDF grids for $n_{f}=5$ flavors with the central value of $\alpha_{s}^{\left(n_{f}=5\right)}\left(M_{Z}\right)$ fixed. The 10 sets cover the range $\alpha_{s}^{\left(n_{f}=5\right)}\left(M_{Z}\right)=0.114 \div 0.123$ with a spacing of 0.001 and are denoted as

ABMP16als114_5_nlo (0+29), 
Table 4 Values of the heavy-quark masses $m_{c}\left(m_{c}\right), m_{b}\left(m_{b}\right)$ and $m_{t}\left(m_{t}\right)$ and $\alpha_{s}^{\left(n_{f}=5\right)}\left(M_{Z}\right)$ in the $\overline{\mathrm{MS}}$ scheme for the PDFs ABMP16_5_nlo $(0+29)$ with $n_{f}=5$. The values for pole masses $m_{b}^{\text {pole }}$ and $m_{t}^{\text {pole }}$ in the on-shell scheme obtained using RunDec [23] are also given

\begin{tabular}{|c|c|c|c|c|c|c|}
\hline $\begin{array}{l}\text { PDF set } \\
0\end{array}$ & $\begin{array}{l}\alpha_{s}^{\left(n_{f}=5\right)}\left(M_{Z}\right) \\
0.11905\end{array}$ & $\begin{array}{l}m_{c}\left(m_{c}\right)[\mathrm{GeV}] \\
1.175\end{array}$ & $\begin{array}{l}m_{b}\left(m_{b}\right)[\mathrm{GeV}] \\
3.880\end{array}$ & $\begin{array}{l}m_{b}^{\text {pole }}[\mathrm{GeV}] \\
4.488\end{array}$ & $\begin{array}{l}m_{t}\left(m_{t}\right)[\mathrm{GeV}] \\
162.08\end{array}$ & $\begin{array}{l}m_{t}^{\text {pole }}[\mathrm{GeV}] \\
171.44\end{array}$ \\
\hline 1 & 0.11905 & 1.175 & 3.880 & 4.488 & 162.08 & 171.44 \\
\hline 2 & 0.11906 & 1.175 & 3.880 & 4.489 & 162.08 & 171.44 \\
\hline 3 & 0.11905 & 1.175 & 3.880 & 4.488 & 162.08 & 171.44 \\
\hline 4 & 0.11899 & 1.175 & 3.880 & 4.488 & 162.08 & 171.43 \\
\hline 5 & 0.11898 & 1.175 & 3.880 & 4.487 & 162.08 & 171.43 \\
\hline 6 & 0.11907 & 1.175 & 3.880 & 4.489 & 162.08 & 171.44 \\
\hline 7 & 0.11906 & 1.175 & 3.880 & 4.489 & 162.08 & 171.44 \\
\hline 8 & 0.11911 & 1.175 & 3.880 & 4.489 & 162.08 & 171.44 \\
\hline 9 & 0.11858 & 1.175 & 3.880 & 4.482 & 162.08 & 171.40 \\
\hline 10 & 0.11925 & 1.175 & 3.880 & 4.491 & 162.08 & 171.46 \\
\hline 11 & 0.11914 & 1.175 & 3.880 & 4.490 & 162.08 & 171.45 \\
\hline 12 & 0.11910 & 1.176 & 3.880 & 4.489 & 162.08 & 171.44 \\
\hline 13 & 0.11904 & 1.173 & 3.880 & 4.488 & 162.08 & 171.44 \\
\hline 14 & 0.11909 & 1.203 & 3.880 & 4.489 & 162.08 & 171.44 \\
\hline 15 & 0.11912 & 1.170 & 3.880 & 4.489 & 162.08 & 171.44 \\
\hline 16 & 0.11930 & 1.169 & 3.877 & 4.489 & 162.08 & 171.46 \\
\hline 17 & 0.11897 & 1.174 & 3.754 & 4.351 & 162.08 & 171.43 \\
\hline 18 & 0.11883 & 1.179 & 3.878 & 4.483 & 162.09 & 171.43 \\
\hline 19 & 0.11904 & 1.175 & 3.884 & 4.493 & 162.08 & 171.44 \\
\hline 20 & 0.11879 & 1.180 & 3.888 & 4.493 & 162.13 & 171.47 \\
\hline 21 & 0.11901 & 1.179 & 3.872 & 4.479 & 162.06 & 171.41 \\
\hline 22 & 0.11914 & 1.180 & 3.882 & 4.492 & 162.05 & 171.41 \\
\hline 23 & 0.11889 & 1.169 & 3.880 & 4.486 & 162.12 & 171.47 \\
\hline 24 & 0.11879 & 1.178 & 3.875 & 4.479 & 161.86 & 171.19 \\
\hline 25 & 0.11980 & 1.169 & 3.881 & 4.500 & 162.97 & 171.44 \\
\hline 26 & 0.11914 & 1.182 & 3.881 & 4.491 & 162.12 & 171.49 \\
\hline 27 & 0.11892 & 1.171 & 3.879 & 4.486 & 161.89 & 171.23 \\
\hline 28 & 0.11888 & 1.176 & 3.882 & 4.488 & 161.88 & 171.21 \\
\hline 29 & 0.11936 & 1.176 & 3.870 & 4.482 & 162.34 & 171.74 \\
\hline
\end{tabular}

\section{ABMP16als115_5_nlo $(0+29)$, ABMP16als116_5_nlo $(0+29)$,

where the value of $\alpha_{S}^{\left(n_{f}=5\right)}\left(M_{Z}\right)$ has been fixed as indicated in the file names. These grids are determined by re-fitting all PDF parameters for the individual choices of $\alpha_{s}$, which for technical consistency remains a formal parameter in the fit, but with greatly suppressed uncertainty.

As the heavy-quark masses $m_{c}\left(m_{c}\right), m_{b}\left(m_{b}\right)$ and $m_{t}\left(m_{t}\right)$ have been fitted their numerical values vary for each of the 29 PDF sets and cross section computations involving heavy quarks have to account for this. For reference we list in Table 4 the heavy-quark masses in the ABMP16 grids and the values of $\alpha_{s}^{\left(n_{f}=5\right)}\left(M_{Z}\right)$. These values can also be easily retrieved within the LHAPDF library framework. The bottom- and the top-quark pole masses, $m_{b}^{\text {pole }}$ and $m_{t}^{\text {pole }}$, which are required for the on-shell scheme are also provided in Table 4. In particular, for computations with the central ABMP16 set at NLO the values $m_{b}^{\text {pole }}=4.488 \mathrm{GeV}$ and $m_{t}^{\text {pole }}=171.44 \mathrm{GeV}$ should be used. ${ }^{2}$

Finally, we also provide the results of the variants with no constraints on the fit parameters, in particular on the higher-

\footnotetext{
$\overline{2}$ In the matching of $m_{c}\left(m_{c}\right)$ to the on-shell scheme $m_{c}^{\text {pole }}$ acquires large QCD corrections up to $\mathrm{N}^{3} \mathrm{LO}$ [14], therefore use of $m_{c}^{\text {pole }}$ is problematic in this context [1].
} 
twist terms, which are extracted at NLO as well. For $n_{f}=$ 3,4 and 5 flavors these are the sets

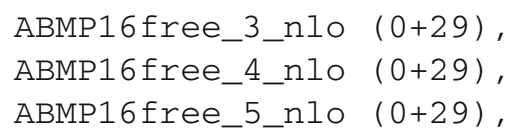

which come, as discussed above, essentially with larger values for $\alpha_{s}\left(M_{Z}\right)$ and $m_{t}\left(m_{t}\right)$, e.g., $\alpha_{s}^{\left(n_{f}=5\right)}\left(M_{Z}\right)=0.1227$ and $m_{t}\left(m_{t}\right)=164.47 \mathrm{GeV}$.

The benchmark cross sections for the Higgs-boson and top-quark pair production at the LHC at NLO and NNLO with consistent use of the PDF sets obtained in the present analysis are given in Table 5 . The quoted errors denote the PDF and $\alpha_{s}$ uncertainties derived from the uncertainties in the experimental data. Thus, they are of similar size at NLO and NNLO.

The Higgs boson cross section $\sigma(H)$ is computed in the effective theory in the limit $m_{t} \rightarrow \infty$, but with full $m_{t}$ dependence in the Born cross section, based on the NNLO results of Refs. [15-17]. The NLO value of $\sigma(H)$ is about $20 \%$ smaller than the NNLO one due to missing large perturbative corrections, which are only partially compensated by a larger value of $\alpha_{s}$. In the effective theory the Born cross section for $\sigma(H)$ is proportional to $\alpha_{s}^{2}$, so that the variant of the NLO fit with the larger value for the strong coupling, $\alpha_{s}^{\mathrm{NLO}}\left(M_{Z}\right)=0.1227$, gives a NLO cross section increased by $5 \%$, i.e. $\sigma(H)=$ $35.2 \pm 0.58 \mathrm{pb}$ with the PDF set ABMP16 free_5_nlo compared to $\sigma(H)=33.59 \pm 0.58 \mathrm{pb}$ with the PDF set ABMP16_5_nlo in Table 5.

The inclusive cross section $\sigma(t \bar{t})$ for top-quark pair production uses Ref. [18] based on Refs. [19-22]. In this case, the NLO and NNLO values of $\sigma(t \bar{t})$ for the range of centerof-mass energies explored at the LHC are similar, since those data have been included in both fits and are accommodated by the corresponding changes in the value of $\alpha_{s}$ and the topquark mass $m_{t}$, cf. Fig. 3 and Table 3.

In summary, we have completed the determination of the ABMP16 PDF sets at those orders of perturbation theory, which are currently of phenomenological relevance, i.e., at NLO and NNLO. Essential input in the ABMP16 analysis has been the final HERA DIS combination data from run I+II, which has consolidated the available world DIS data. In addition, several new data sets from the fixed-target DIS together with recent LHC and Tevatron data for the DY process and for the top-quark hadro-production have been used.

We have discussed the features of the NLO extraction of PDFs, which in general, have a few limitations due to lacking constraints of the higher order Wilson coefficients and we have emphasized the consistent use of PDFs and an order-dependent value of $\alpha_{s}\left(M_{Z}\right)$, which is absolutely crucial because of correlations. The same holds, to a lesser 
extent, in collider processes also for the values of the heavyquark masses used.

The ABMP16 PDFs establish the baseline for high precision analyses of LHC data from run I and run II, and the NLO variant is now available for computing cross sections of scattering processes with multi-particle final states, for which the NNLO QCD corrections will not be available in the foreseeable future, or for Monte Carlo studies. Precision analyses of LHC data, however, will always require analyses to NNLO accuracy in QCD. This will become even more important with the arrival of the data from the high luminosity runs.

Acknowledgements We would like to thank K. Lipka and O. Zeniaev for cross-checks of the preliminary version of the LHAPDF grids derived from this analysis. This work has been supported by Bundesministerium für Bildung und Forschung (Contract 05H15GUCC1).

Open Access This article is distributed under the terms of the Creative Commons Attribution 4.0 International License (http://creativecomm ons.org/licenses/by/4.0/), which permits unrestricted use, distribution, and reproduction in any medium, provided you give appropriate credit to the original author(s) and the source, provide a link to the Creative Commons license, and indicate if changes were made.

Funded by SCOAP ${ }^{3}$.

\section{References}

1. A. Accardi et al., Eur. Phys. J. C 76, 471 (2016). arXiv: 1603.08906

2. S. Alekhin, J. Blümlein, S. Moch, Phys. Rev. D 86, 054009 (2012). arXiv: 1202.2281

3. S. Alekhin, J. Blümlein, S. Moch, Phys. Rev. D 89, 054028 (2014). arXiv: 1310.3059

4. S. Alekhin, J. Blümlein, S. Moch, R. Placakyte, Phys. Rev. D 96, 014011 (2017). arXiv: 1701.05838

5. A. Buckley et al., Eur. Phys. J. C 75, 132 (2015). arXiv: 1412.7420

6. J. Blümlein, S. Riemersma, W.L. van Neerven, A. Vogt, Nucl. Phys. Proc. Suppl. 51C, 97 (1996). arXiv: hep-ph/9609217

7. S. Alekhin, J. Blümlein, S.O. Moch, Mod. Phys. Lett. A 31(25), 1630023 (2016)
8. H1 Collaboration, V. Andreev et al., Eur. Phys. J. C 77(11), 791 (2017) arXiv: 1709.07251

9. J. Rojo et al., J. Phys. G42, 103103 (2015). arXiv: 1507.00556

10. S. Dulat et al., Phys. Rev. D 93, 033006 (2016). arXiv: 1506.07443

11. NNPDF, R. D. Ball et al., Eur. Phys. J. C77, 663 (2017), arXiv: 1706.00428

12. K. Prytz, Phys. Lett. B 311, 286 (1993)

13. L.A. Harland-Lang, A.D. Martin, P. Motylinski, R.S. Thorne, Eur. Phys. J. C 76(1), 10 (2016). arXiv: 1510.02332

14. P. Marquard, A.V. Smirnov, V.A. Smirnov, M. Steinhauser, Phys. Rev. Lett. 114, 142002 (2015). arXiv: 1502.01030

15. R.V. Harlander, W.B. Kilgore, Phys. Rev. Lett. 88, 201801 (2002). arXiv: hep-ph/0201206

16. C. Anastasiou, K. Melnikov, Nucl. Phys. B 646, 220 (2002). arXiv: hep-ph/0207004

17. V. Ravindran, J. Smith, W.L. van Neerven, Nucl. Phys. B 665, 325 (2003). arXiv: hep-ph/0302135

18. M. Aliev et al., Comput. Phys. Commun. 182, 1034 (2011). arXiv: 1007.1327

19. P. Bärnreuther, M. Czakon, A. Mitov, Phys. Rev. Lett. 109, 132001 (2012). arXiv: 1204.5201

20. M. Czakon, A. Mitov, JHEP 12, 054 (2012). arXiv: 1207.0236

21. M. Czakon, A. Mitov, JHEP 01, 080 (2013). arXiv: 1210.6832

22. M. Czakon, P. Fiedler, A. Mitov, Phys. Rev. Lett. 110, 252004 (2013). arXiv: 1303.6254

23. K.G. Chetyrkin, J.H. Kühn, M. Steinhauser, Comput. Phys. Commun. 133, 43 (2000). arXiv: hep-ph/0004189 